3.

\title{
Ein neues Verfahren, die Nasen-Rachenhöhle mit ihren pneumatischen Anhängen am Leichnam ohne äussere Entstellung freizulegen.
}

\author{
Von Dr. Th. Harke in Hamburg.
}

Die Erkrankungen im Gebiete der Nasenhöhle und ihrer pneumatischen Anhänge machen es für den Kliniker wünschenswertb, dass die anatomische Besichtigung dieser Organe nicht nur auf die relativ geringe Anzahl der Leichen beschränkt wird, welche den anatomischen Instituten zur vollständigen Zergliederung zur Verfügung steben, sondern dass dieselbe überall da ausgeführt werden kann, wo es klinische Interessen erfordern und ein grosses Leichenmaterial zur Verfügung steht, eine äussere Entstellung der Leiche aber mit Rücksicht auf die Bestattung zu vermeiden ist.

Schalle') hat eine Sectionsmethode für die Nasen-Rachen- und Gehörorgane angegeben, we]che gestattet, dieselben ohne äussere Entstellung aus dem Kopfe mit oder ohne Zusammenbang wit den Mund- und Halseingeweiden herauszunehmen. Es ist dies, wie Zuckerkandl ${ }^{2}$ ) in seiner Anatomie der Nasenböhle betont, die einzige für solche Leichen, welcbe auf dem Todtenbette nicht entstellt sein dürfen.

Der Schalle'sche Schnitt hat grosse Vorzüge, welche besonders darin bestehen, dass das Präparat auch die Gebörorgane entbält, und dass es, nachdem man nach der Herausnahme des ganzen Präparats aus dem Schädel die bintere Wand der Speiseröhre und des Rachens durebsehnitten hat, das natürliche Bild des Nasenrachenraumes und Kehlkopfeinganges bietet und conservirt werden kann. Auf der anderen Seite hat er grosse Mängel und entschiedene Nachtheile, welche erstere darin besteben, dass diese Sectionsmethode trotz der gegentbeiligen Angaben des Autors zeitraubend und ohne Hülfe allein nicht leicht ausführbar ist, auch ein complicirtes Instrumentariun, welches nicht überall vorbanden ist, erfordert; zudem ist die Technik recht complicirt und nicht unmittelbar dureh die Kenntniss der Anatomie plausibel. Die grossen Nachtheile aber bringt die Sectionsmethode mit sich, dass die Organe in den Nasenhöhlen durch den durch dieselben frontal verlaufenden Sägeschnitt so durchtrennt werden, dass man niemals die vorderen knden der mittleren und unteren Muscheln im Präparat erhält und fast immer die Gegend des Infundibulum zerstört wird; dies fällt aber um so mehr in's Gewicht, als gerade bier so bäufig pathologiscbe Prozesse vorkommen.

Da eine bessere Methode bisher nicht gegeben war, so musste man diese grossen Nachtheile mit in den. Kauf nehmen.

1) Schalle, Dieses Archiv. Bd. 71 .

2) Zuckerkandl, Normale und pathologische Anatomie der Nasenhöhle und ibrer pneumat. Anhänge. Wien 1882. 
Fig. 1.

Laterale Wand der Nasenhöhle mit entfernter zweiter Muschel, um die Communicationsöffnungen mit den pneumatischen Nebenräumen zu zeigen, nach Henle.

.... Schnittebene der Säge beim Schalle'schen Schnitt.

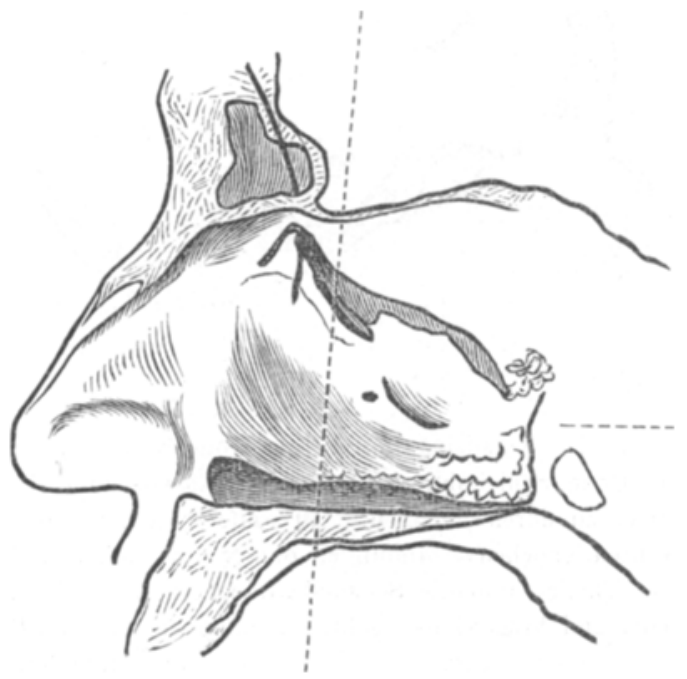

Für die Fälle, wo man das Hauptgewicht auf die Besichtigung der Nasenhöhlen mit ibren pneumatischen Anbängen und des Nasenrachenraumes legt, die Gehörorgane jedoch kein hervorragendes Interesse erfordern, möchte ich ein Verfahren vorschlagen, welches ohne grossen Zeitaufwand mit dem gewöhnlichen Sections-Instrumentarium ohne Kenntniss einer complicirten Techuik ausgeführt werden kann und obne äussere Entstellung die ganzen Nasenböblen mit ihren pneumatischen Anhängen und den Nasenrachenraum zur Besichtigung freilegt. - Man erreicht dies wit demselben Sagittalschnitte, welcher schon auf dem Präparirsaal zur Demonstration der Nasenböhlen angewandt wird, mit geringen Einschränkungen, um eine åussere Entstellung der Leichen zu vermeiden.

Nachdem das Gehirn in der üblichen Weise der Schädelhöhle entnommen ist, wird die Kopfhaut vorn und hinten so tief wie möglich vom Schädel abpräparirt, so dass die Augenbogen und Nasenbeine und das Binterhauptsloch frei werden; sodann wird die Nackenmusculatur vom Scbädel abgeschnitten. - Nach passender Lagerung wird nun, je nachdem man die rechte oder linke Nasenhöhle zuerst besichtigen will, ein Sagittalschnitt etwa $0,3 \mathrm{~cm}$ von der Medianebene des Schädels nach rechts oder links mit der Knochensäge geführt, bis man den Schädel vorn bis auf die Nasenbeine, hinten bis in's Binterhauptsloch durehsägt bat. Wie an einem Durehschnitt zu sehen ist, wird man hierdureb die Schädelbasis fast überall durebtrennt haben; noch nicht durchschnittene Knochenbrücken und der Zahn des Epistropheus werden nachtrăglich mit einer Stichsäge durchsägt. Jetzt bilden noch die Nasenbeine, der harte Gaumen, der Zahnfortsatz des Oberkiefers feste Knochenverbindungen der beiden Schädelbälften; dieselben gestatten ein Auseinander- 
Eig. 2.

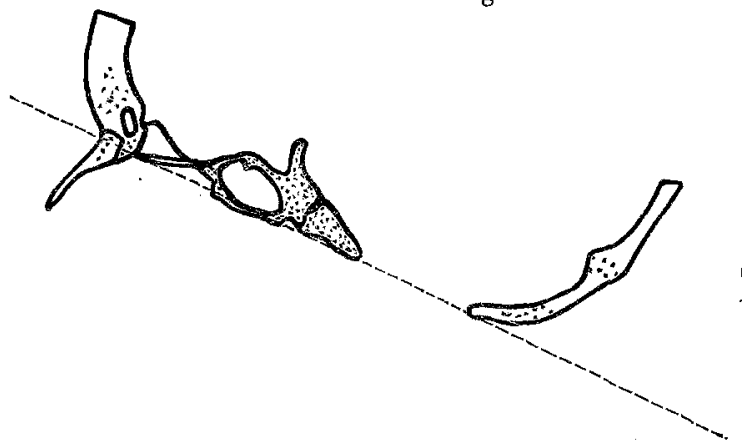

Fast medianer

Durchschnitt durch den Schädel nach Henle.

Zeigt, wie weit die Säge vorn und hinten eindringen darf.

Dr. Harke.

biegen der Schädelhälften in genügender Breite, um bisher nicht durchschnittene Schleimbautüberzüge des Nasen- und Rachendaches mit einem Messer zu durchschneiden. Auf weiteres Auseinanderbiegen brechen die genannten Knochenverbindungen ohne Schwierigkeit und ohne Zerstörnng wichtiger Theile, und die Schädelbälften rotiren um je eine Axe, welche durch Kiefer und Atlasgelenke geht, und klappen nach der Art eines Buches weiter

Fig. 3.

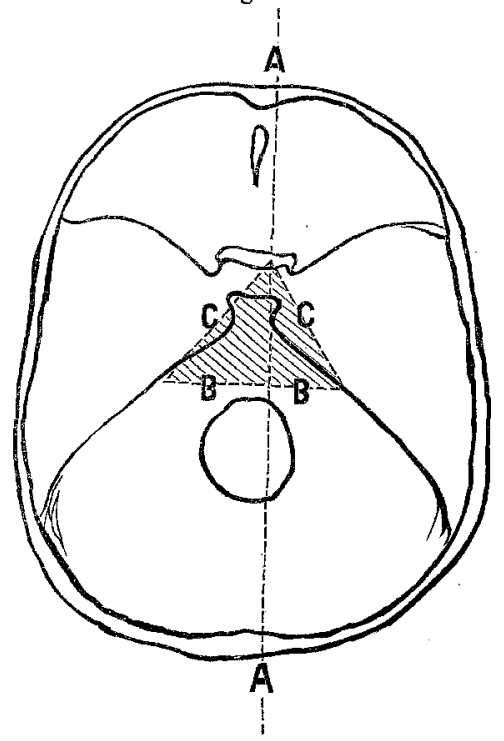

Schädelbasis mit den Grenzlinien der Schädelgruben nach Henle.

A A Sagittalsehnitt rechts von der Mittellinie. B B mit der Sticbsäge geführte Schnitte. C C desgl. aus einander. In vielen Fällen ist schon jetzt der Einblick in die zuerst eröffnete Nasenböble ein genügender, und nach Abtragung des Septums Jässt sich auch die andere Nasenhöble äbersehen. - Ist dies nicht der Fall, so sägt man mit der Stichsäge die Scbädelbasis senkrecht zum Sagittalschnitt rechts und links an der Stelle durch, wo die obere Wand des $\mathrm{Na}$ senrachenraumes in die hintere äbergeht, und zwar soweit, bis man an die Seitenwand des Nasenrachenraumes rechts und links gelangt. Ferner führt man von dem Punkte, wo der Sagittalschnitt den Türkensattel durchschneidet, einen Sägeschnitt mit der Stichsäge nach dem Endpunkte des zuerst beschriebenen hin, so dass man nunmehr jederseits aus der Schädelbasis ein rechtwinkliges Dreieck herausgesägt bat. Nachdem durch den Sagittalschnitt die Keilbeinhöhle der betreffenden Seite, auf welcher der Sagittalschnitt verlief, eröffnet war, wird durch den letatbeschriebenen 
Stichsägeschnitt die Keilbeinhöhle der anderen Seite eröffnet. Nachdem die ausgesägten Stücke der Schädelbasis herausgenommen sind, hat man stets einen breiten Einblick in die Nasenböhlen.

In der zunächst eröffneten Nasenböhle betrachtet man durch successive Abtragung der Muscheln mit Messer und Cooper'scher Scheere die Gegend des Infundibulum und die Nasenseitenwand; dureb Resection eines Theils der dünnen Seitenwand erlangt man einen Einblick in die Oberkieferböble. Die Abtragung der Nasenscheidewand erlaubt die Besichtigung der anderen Nasenhöhle in ähnlicher Weise. Stirnhöblen und Siebbeinzellen lassen sich, soweit sie noch nicht eröffnet sind, einfach eröffuen.

Das Verfabren ist bei Leichen jeden Alters obne äussere Entstellung auszufübren.

Wenn erwünscht, kann die Besichtigung der Gehörorgane nach Schalle oder einer sonstigen Methode angescblossen werden.

Ich glaube, dass das von mir angegebene Verfahren wegen seiner Einfachheit und unbeschränkten Zulässigkeit die Besichtigung der Nasenhöhlen bedeutend bäufiger machen und so dem klinischen Interesse, welches auf diesem Gebiete bisher wenig befriedigt werden konute, in erwünschter Weise gerecht werden wird.

4.

\section{Versammlung der Gesellschaft deutscher Naturforscher und Aerzte.}

Die 64. Versammlung der Gesellschaft deutscher Naturforscher und Aerzte wird, gemäss dem Beschlusse der vorjährigen Versammlung zu Bremen, voin 21.-25. September d. J. in Halle a. S. tagen.

Obwohl die Versammlung nach den Statuten eine Gesellschaft deutscher Naturforscher und Aerzte ist, so ist doch die Betheiligung fremder Gelehrten stets im hohen Grade willkommen geheissen worden; dieselben werden zur Betheiligung an den Arbeiten der Versammlung freundlichst eingeladen.

Jeder Theilnehmer an der Versammlung entrichtet einen Beitrag von $12 \mathrm{M}$. und erhält dafür eine Festkarte, ein Abzeichen und die für die Versammlung bestimmten Druckschriften; zugleich erwirbt er damit Anspruch auf die Lösung von Damen-Festkarten zum Preise von 6 M.

Bei der Beratbung und Beschlussfassung über die Angelegenheiten der Gesellschaft deutscher Naturforscher und Aerzte sind nur die Mitglieder dieser Gesellschaft, welche ausser dem Theilnehmerbeitrag noch einen Jahresbeitrag von $5 \mathrm{M}$. zu entrichten haben, stimmberechtigt. Das Stimmrecht wird vermittelst der von dem Schatzmeister ausgegebenen Mitgliedskarten ausgeübt.

Die drei allgemeinen Sitzungen werden in dem grossen Saale der "Kaisersäle", die Sitzungen der Abtheilungen in den Hörsälen des Universitätsgebäudes und der Universitätsinstitute stattfinden.

Nach Beendigung der zweiten allgemeinen Sitzung am 23. September wird eine Geschäftssitzung der Gesellschaft behufs Berathung und Beschlussfassung über einen, von dem Vorstande ausgearbeiteten anderweiten Entwurf zu Gesellschaftsstatuten abgehalten werden. Einen Abdruck der Vorschläge des Vorstandes fügen wir gleichfalls hier bei.

Eine allgemeine Ausstellung wissenschaftlicher Apparate, Instrumente und Präparate wird diesmal mit der Versammlung nicht verbunden sein. Dagegen wird die allgemeine Elektricitäts-Gesellschaft eine kleine Ausstellung elektrotechnischer und elektrolytischer Gegenstände in der städ- 